\title{
Airway management of an ankylosing spondylitis patient with severe temporomandibular joint ankylosis and impossible mouth opening
}

\author{
Jong-Man Kang, Kang-Woo Lee, Dong-Ok Kim, and Jae-Woo Yi
}

Department of Anesthesiology and Pain Medicine, Kyung Hee University Hospital at Gangdong, College of Medicine, Kyung Hee University, Seoul, Korea

In most cases of surgery requiring general anesthesia, airway management and endotracheal intubation are important procedures that must take place. However, when a patient has ankylosing spondylitis (AS) involving the cervical spine in addition to a temporomandibular joint ankylosis (TMJA), head manipulation is difficult to perform. Also, when a patient has limited mouth opening or is unable to open the mouth altogether, airway management and endotracheal intubation are significantly difficult. We experienced the case of an AS patient who was completely unable to open the mouth due to TMJA, admitted for osteotomy and osteoplasty under general anesthesia.

A 34-year-old man $(44.7 \mathrm{~kg}, 132.7 \mathrm{~cm})$ with TMJA was admitted to the Oral and Maxillofacial Surgery Department to receive surgery for the mouth opening disorder. The patient was scheduled to receive corrective surgery for the secondary spinal transformation due to AS and had severe kyphosis in the thoracic and lumbar spine due to complete AS (Fig. 1A). In 1997, the patient underwent a cervical spine u-bar insertion and temporomandibular joint surgery at Cedars-Sinai Medical Center, US. (Fig. 1B). A tracheostomy was also performed which left the patient with a surgical scar on his left neck. At the time of the surgery in 1997, the mouth opening was about $25 \mathrm{~mm}$ but several years following the procedure, abnormal coronoid process hypertrophy and TMJA (Fig. 1C) developed. For more than 10 years following the disease development, the patient could not tolerate a solid diet but only a liquid diet such as milk or soy milk. When the patient was finally assessed during this admission, his ability to open his mouth was completely impossible (Fig. 1D).

An anesthetic induction was performed by an anesthesiologist with over 200 awake fiberoptic bronchoscopic intubation experiences. Glycopyrrolate $0.2 \mathrm{mg}$ was given as premedication, and $4 \%$ lidocaine was injected trans-tracheally to anaesthetise the trachea. To prevent bleeding from the nasal cavity, gauze soaked with $0.1 \%$ epinephrine for 15 minutes was packed in the nasal cavity then, a lubricated $5 \mathrm{~mm}$ diameter fiberoptic bronchoscope (LF-TP, Olympus Optical Company, Tokyo, Japan) was passed through the right nostril to confirm the airway. After spraying $4 \mathrm{ml}$ of $4 \%$ lidocaine on the vocal cord for 15 seconds, the end of the fiberoptic bronchoscope was passed through the vocal cords, and positioned between the vocal cords and carina. Afterwards, an endotracheal tube was placed. After confirming endotracheal intubation, anesthetic induction was conducted using propofol $90 \mathrm{mg}$ and rocuronium 50 mg. Although the tracheostomy was expected to be difficult due to the limited cervical spine movement and the history of tracheostomy, a tracheostomy set and a jet ventilator were prepared to cope with the emergency situation in airway control during the whole procedure.

For anesthesia maintenance, $2 \%$ propofol and remifentanil were administered using a computer-controlled infusion pump

Corresponding author: Jae-Woo Yi, M.D., Ph.D., Department of Anesthesiology and Pain Medicine, Kyung Hee University Hospital at Gangdong, College of Medicine, Kyung Hee University, 149, Sangil-dong, Gangdong-gu, Seoul 134-727, Korea. Tel: 82-2-440-6192, Fax: 82-2440-7808, E-mail: mdyjwchk@khu.ac.kr

(c) This is an open-access article distributed under the terms of the Creative Commons Attribution Non-Commercial License (http:// creativecommons.org/licenses/by-nc/3.0/), which permits unrestricted non-commercial use, distribution, and reproduction in any medium, provided the original work is properly cited. 

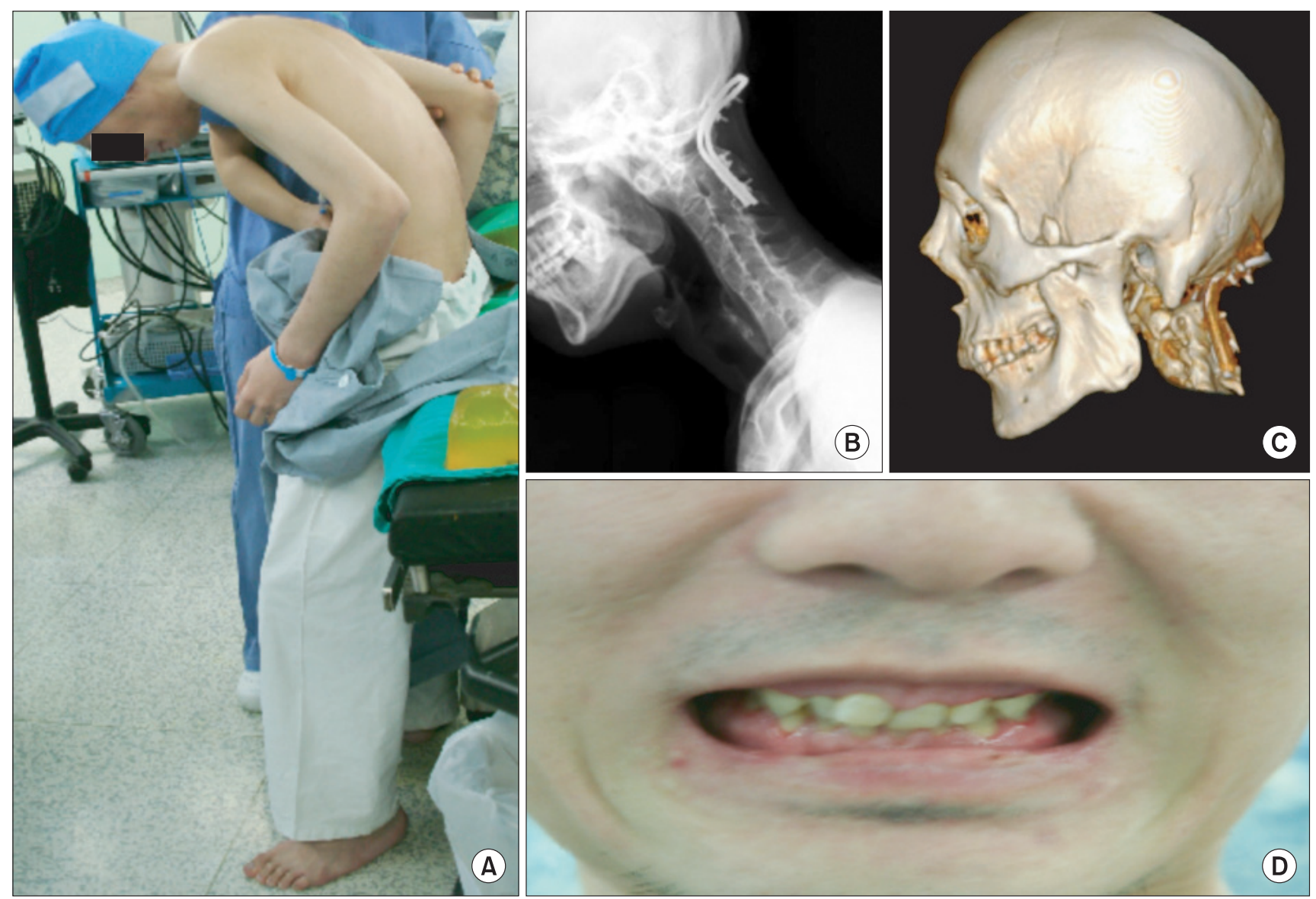

Fig. 1. (A) In this picture, the patient is in a preoperative upright position in which there is severe whole spine kyphosis due to complete ankylosing spondylitis. The plane X-ray of the neck (B) and 3D CT of the skull (C) show the inserted u-bar to cervical spine and the ankylosis of both temporomandibular joints with the abnormal coronoid process hypertrophy. (D) This image shows the mouth that is impossible to open.

(Orchestra ${ }^{\circledR}$ Base Primea, Fresenius Vial, France). During the surgery, the patient remained in a semi-fowlers position, had an EKG within normal range with the blood pressure of 13580/90-50 mmHg, heart rate of 50-102 beats per minute, and SpO2 at $100 \%$. The operation took 10 hours and 15 minutes. In order to prevent airway obstruction caused by the swelling of the airway, the patient was not extubated and was transferred to the intensive care unit while sedated using dexmedetomidine. At postoperative day 1, a lack of airway swelling and selfrespiration was confirmed then an extubation was carefully conducted before the patient was transferred to the general ward.

At postoperative day-12, the patient was confirmed to have no problems in airway management, and was discharged. At the time of discharge, the maximum mouth opening was 30 $\mathrm{mm}$. During the hospital stay, no particular problem in airway management was observed. Currently the patient is able to tolerate small amounts of solid foods, and is on physical rehabilitation.
In cases where endotracheal intubation using conventional direct laryngoscope is impossible, procedures such as fiberoptic bronchoscopic intubation, retrograde tracheal intubation or tracheostomy may be performed $[1,2]$. When endotracheal intubation is expected to be difficult due to AS involving the cervical spine, and flexion, extension and rotation of the cervical region are not possible, intubation using noninvasive video laryngoscope such as Glidescope video laryngoscope (GVL, Verathon Inc., Bothell, USA), Pentax-AWS (Pentax Corporation, Tokyo, Japan) and Airtraq optical laryngoscope (Airtraq, Prodol Meditec, Vizcaya, Spain) or fiberoptic bronchoscope should be considered. However, if the patient is accompanied by TMJA, and is not able to open their mouth, use of a video laryngoscope will be impossible, thus fiberoptic bronchoscopic intubation is restricted to the nasotracheal approach. Cases of performing retrograde tracheal intubation on AS patients were reported [3], but this procedure can be hardly applied to patients who are absolutely unable to open the mouth. Additionally, if bleeding caused by guide wire arises, fiberoptic bronchoscopic 
intubation would be difficult, as well.

The incidence rate of epistaxis is considerably high at $19-54 \%$ in nasotracheal intubation $[4,5]$ and when epistaxis develops, a view of the fiberoptic bronchoscope can be compromised even by a small amount of bleeding and consequently, endotracheal intubation may be delayed or a risk of hypoxia may increase. Moreover, in patient cases, such as the present case, that has abnormal positioning and impossible mouth opening, the development of epistaxis under conditions of unawareness may result in difficult ventilation, and an increased risk of pulmonary aspiration and accordingly, success of endotracheal intubation cannot be guaranteed. Also, since the triple airway maneuver is not applicable to TMJA, risk of developing airway obstruction caused by the tongue increases under general anesthesia and accordingly, ventilation can hardly be maintained. When fiberoptic bronchoscopic nasotracheal intubation is conducted under conditions of awareness, the risk of developing hypoxia may be reduced, and pulmonary aspiration due to epistaxis may also be prevented and accordingly, the necessity to perform a tracheostomy may be reduced.

In conclusion, in case of TMJA with a high risk of airway control due to impossible mouth opening and limited cervical spine mobility, skillful fiberoptic bronchoscopic nasotracheal intubation under conditions of awareness should be considered a safe procedure.

\section{References}

1. Kim HJ, Rhee KY, Yum KW. Nasotracheal intubation using fiberoptic bronchoscope and guidewire in a pediatric mandibular fracture patient. Korean J Anesthesiol 1999; 36: 162-4.

2. Barriot P, Riou B. Retrograde technique for tracheal intubation in trauma patient. Crit Care Med 1988; 16: 712-3.

3. Raval C, Patel H, Patel P, Kharod U. Retrograde intubation in a case of ankylosing spondylitis posted for correction of deformity of spine. Saudi J Anaesth 2010; 4: 38-41.

4. Watanabe S, Yaguchi Y, Suga A, Asakura N. A "bubble-tip" (Airguide) tracheal tube system: its effects on incidence of epistaxis and ease of tube advancement in the subglottic region during nasotracheal intubation. Anesth Analg 1994; 78: 1140-3.

5. Morimoto Y, Sugimura M, Hirose Y, Taki K, Niwa H. Nasotracheal intubation under curve-tipped suction catheter guidance reduces epistaxis. Can J Anaesth 2006; 53: 295-8. 